\title{
Accuracy and reproducibility of simplified QSPECT dosimetry for personalized ${ }^{177} \mathrm{Lu}$ octreotate PRRT
}

Michela Del Prete ${ }^{1,2}$, Frédéric Arsenault ${ }^{1,2}$, Nassim Saighi ${ }^{1,2}$, Wei Zhao ${ }^{3,4}$, François-Alexandre Buteau ${ }^{1,2}$, Anna Celler ${ }^{3,4}$ and Jean-Mathieu Beauregard ${ }^{1,2^{*}}$ (i)

* Correspondence: jean-mathieu. beauregard@chudequebec.ca ${ }^{1}$ Department of Radiology and Nuclear Medicine and Cancer Research Center, Université Laval, Quebec City, Canada

${ }^{2}$ Department of Medical Imaging and Oncology Branch of $\mathrm{CHU}$ de Québec Research Center, CHU de Québec - Université Laval, 11 côte du Palais, Quebec City, QC G1R 2J6, Canada

Full list of author information is available at the end of the article

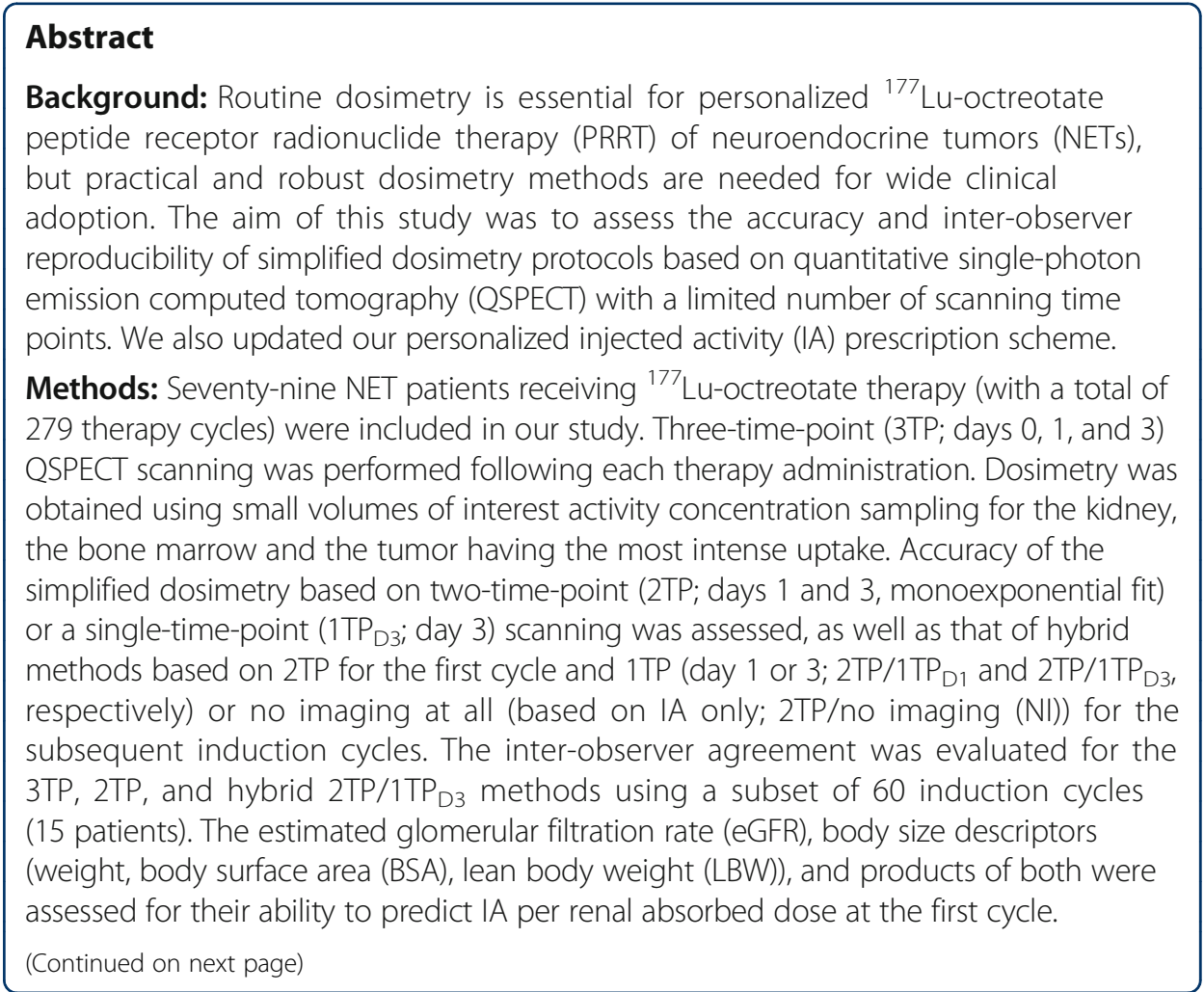

(c) The Author(s). 2018 Open Access This article is distributed under the terms of the Creative Commons Attribution 4.0 Internationa License (http://creativecommons.org/licenses/by/4.0/), which permits unrestricted use, distribution, and reproduction in any medium, provided you give appropriate credit to the original author(s) and the source, provide a link to the Creative Commons license, and indicate if changes were made. 
(Continued from previous page)

Results: The 2TP dosimetry estimates correlated highly with those from the 3TP data for all tissues (Spearman $r>0.99, P<0.0001$ ) with small relative errors between the methods, particularly for the kidney and the tumor, with median relative errors not exceeding $2 \%$ and interdecile ranges spanning over less than $6 \%$ and $4 \%$, respectively, for the per-cycle and cumulative estimates. For the bone marrow, the errors were slightly greater (median errors $<6 \%$, interdecile ranges $<14 \%$ ). Overall, the strength of correlations of the absorbed dose estimates from the simplified methods with those from the 3TP scans tended to progressively decrease, and the relative errors to increase, in the following order: $2 T P, 2 T P / 1 T P_{D_{3}}, 1 T P_{D_{3}}, 2 T P / 1 T P_{D 1}$, and 2TP/NI. For the tumor, the 2TP/NI scenario was highly inaccurate due to the interference of the therapeutic response. There was an excellent inter-observer agreement between the three observers, in particular for the renal absorbed dose estimated using the 3TP and 2TP methods, with mean errors lesser than $1 \%$ and standard deviations of $5 \%$ or lower. The eGFR - LBW and eGFR . BSA products best predicted the ratio of IA to the renal dose (GBq/Gy) for the first cycle (Spearman $r=0.41$ and 0.39 , respectively; $P<0.001$ ). For the first cycle, the personalized IA proportional to eGFR - LBW or eGFR - BSA decreased the range of delivered renal absorbed dose between patients as compared with the fixed IA. For the subsequent cycles, the optimal personalized IA could be determined based on the prior cycle renal GBq/Gy with an error of less than $21 \%$ in $90 \%$ of patients.

Conclusions: A simplified dosimetry protocol based on two-time-point QSPECT scanning on days 1 and 3 post-PRRT provides reproducible and more accurate dose estimates than the techniques relying on a single time point for non-initial or all cycles and results in limited patient inconvenience as compared to protocols involving scanning at later time points. Renal absorbed dose over the 4-cycle induction PRRT course can be standardized by personalizing IA based on the product of eGFR with LBW or BSA for the first cycle and on prior renal dosimetry for the subsequent cycles.

Keywords: Dosimetry, Neuroendocrine tumors, Peptide receptor radionuclide therapy, Personalized, Quantitative SPECT

\section{Background}

For patients with metastatic neuroendocrine tumors (NETs), peptide receptor radionuclide therapy (PRRT) with ${ }^{177} \mathrm{Lu}$-octreotate is an effective palliative treatment that rarely causes serious toxicity $[1,2]$. PRRT has been mostly administered as a 4-cycle induction course using a fixed injected activity (IA) of not more than 7.4 GBq per cycle, in order to not exceed cumulative absorbed doses of 23 Gy to the kidney and 2 Gy to the bone marrow (BM) in the majority of patients [1-4]. However, it is well known that for these critical organs, and in particular for the kidney which is the dose-limiting organ for most patients, the absorbed dose per IA is highly variable and usually lower than 23 Gy per 4 cycles, resulting in most patients being undertreated with such an empiric PRRT regime $[5,6]$. We and others have proposed personalized PRRT (P-PRRT) protocols in which the number of fixed IA cycles or the IA per cycle are modulated to deliver a safe prescribed renal absorbed dose, with the aim to maximize tumor irradiation while keeping the toxicity low $[4,6]$. Such P-PRRT protocols require careful dosimetry monitoring, which is often perceived as a complex and resource-consuming process, therefore constituting a barrier for wide clinical adoption. As a result, the clinical practice of "one-size-fits-all" PRRT prevails, at the potential cost of delivering a suboptimal treatment to most patients. 
We have been routinely performing post-PRRT dosimetry using quantitative single-photon emission computed tomography (QSPECT) combined with the small-sphere volume of interest (VOI) activity concentration sampling [5, 6]. Aiming to simplify the dosimetry process and to reduce the clinical burden thereof, we examined the impact of reducing the number of QSPECT sessions on the accuracy and the inter-observer reproducibility of the resulting dose estimates. In parallel, based on a large dataset from our growing cohort of patients treated with PRRT, we updated our personalized IA determination scheme.

\section{Methods}

Patients and PRRT cycles

From November 2012 to December 2017, 81 patients with progressive metastatic and/ or symptomatic NET were treated with PRRT at CHU de Québec-Université Laval. Two patients who underwent only 1 cycle were each excluded because of their incomplete dosimetric data, and therefore, only data from 79 patients was analyzed. This includes 23 patients who received only empiric PRRT (i.e., fixed IA of approximately $8 \mathrm{GBq}$, occasionally reduced) until March 2016, for whom the requirement for consent was waived due to the retrospective nature of the analysis. All other patients were enrolled in our P-PRRT trial (NCT02754297) and gave informed consent to participate (protocol described in [6]). Patient characteristics are reported in Table 1.

Two hundred and eighty-four therapy cycles were administered during the study period. Five cycles in five patients were excluded from the analysis because of dosimetry protocol deviation or missing data. Among the 279 therapy cycles analyzed, 142 were empiric (median IA = 7.6 GBq; range, 3.8-9.1 GBq) and 137 were personalized (median IA = 9.0 GBq; range, 0.7-32.4 GBq). Anti-nausea premedication (ondansetron and dexamethasone) and a nephroprotective amino acid solution (lysine and arginine) were administered [1]. We administer a 4-cycle induction course for which the prescribed cumulative renal dose is 23 Gy ( 5 Gy at the first cycle; two-monthly intervals) and, in responders only, we offer consolidation, maintenance, and/or salvage cycles (prescribed renal dose of 6 Gy each; personalized intervals). As previously described, prescribed renal absorbed radiation doses were reduced in patients with renal or bone marrow impairment [6].

\section{Reference dosimetry method}

At each cycle, after therapeutic administration of ${ }^{177} \mathrm{Lu}$-octreotate, QSPECT/computed tomography (QSPECT/CT) scans were performed at approximately $4 \mathrm{~h}$ (day 0), $24 \mathrm{~h}$ (day 1), and $72 \mathrm{~h}$ (day 3) using a Symbia T6 camera (Siemens Healthcare, Erlangen, Germany) (Fig. 1) [6, 7]. Following the same data processing as described in [7], the dead-time corrected reconstructed images were converted into the positron emission tomography (PET) DICOM format, which includes a "rescale slope" parameter that converts count data into $\mathrm{Bq} / \mathrm{mL}$ and also enables display of QSPECT images in standardized uptake values normalized for body weight (standardized uptake value; SUV).

These three imaging time points were initially selected for the following practical reasons: (1) the day 0 scan does not incur any additional hospital visit for the patient and allows capturing early kinetics of the radiopharmaceutical and (2) 
Table 1 Patient characteristics

\begin{tabular}{|c|c|}
\hline & All patients $(n=79)$ \\
\hline \multicolumn{2}{|l|}{ Gender, n (\%) } \\
\hline Female & $36(45.6)$ \\
\hline Male & $43(54.4)$ \\
\hline Age at first cycle, median (range) & $60.7(26.1-82.3)$ \\
\hline \multicolumn{2}{|l|}{ Site of primary tumor, $n$ (\%) } \\
\hline Small intestine & $30(38.0)$ \\
\hline Pancreas & $26(32.9)$ \\
\hline Adrenal gland ${ }^{a}$ & $6(7.6)$ \\
\hline Lung & $6(7.6)$ \\
\hline Colon & $2(2.5)$ \\
\hline Stomach & $1(1.3)$ \\
\hline Esthesioneuroblastoma & $1(1.3)$ \\
\hline Unknown & $7(8.9)$ \\
\hline \multicolumn{2}{|l|}{ Metastases, $n(\%)$} \\
\hline Liver & $66(83.5)$ \\
\hline Lymph nodes & $51(64.6)$ \\
\hline Bone & $29(36.7)$ \\
\hline Lung & $9(11.4)$ \\
\hline Other $^{\mathrm{b}}$ & 25 (31.6) \\
\hline \multicolumn{2}{|l|}{ Body size descriptors, mean \pm SD (range) } \\
\hline Weight (Kg) & $72.1 \pm 16.6(42.6-121.0)$ \\
\hline Lean body weight $(\mathrm{Kg})$ & $52.4 \pm 9.9(35.4-81.2)$ \\
\hline Body surface area $\left(m^{2}\right)$ & $1.8 \pm 80.2(1.4-2.5)$ \\
\hline eGFR $\left(\mathrm{ml} / \mathrm{min} / 1.73 \mathrm{~m}^{2}\right)$, mean $\pm \mathrm{SD}$ (range) & $86.3 \pm 22.2(42.0-154.1)$ \\
\hline \multicolumn{2}{|l|}{ Number of cycles, $n(\%)$} \\
\hline 1 & $8(10.1)$ \\
\hline 2 & $6(7.6)$ \\
\hline 3 & $16(20.3)$ \\
\hline 4 & $38(48.1)$ \\
\hline 5 & $3(3.8)$ \\
\hline 6 & $6(7.6)$ \\
\hline 7 & $1(1.3)$ \\
\hline 8 & $1(1.3)$ \\
\hline \multicolumn{2}{|l|}{ Type of cycles, $n$ (\%) } \\
\hline Empiric only & $23(29.1)$ \\
\hline Personalized only & $45(57.0)$ \\
\hline Mixed & $11(13.9)$ \\
\hline
\end{tabular}

eGFR estimated glomerular filtration rate, PRRT peptide receptor radionuclide therapy

${ }^{a}$ Three patients with pheochromocytoma and three patients with paraganglioma

beritoneum, ovary, subcutaneous, pleura, meninges

performing scans beyond day 3 would not be easy for logistical reasons (PRRT being administered on Tuesday, day 4 or 5 would fall on the weekend) and would inconvenience patients (in particular those out-of-city patients who would need to prolong their stay). 


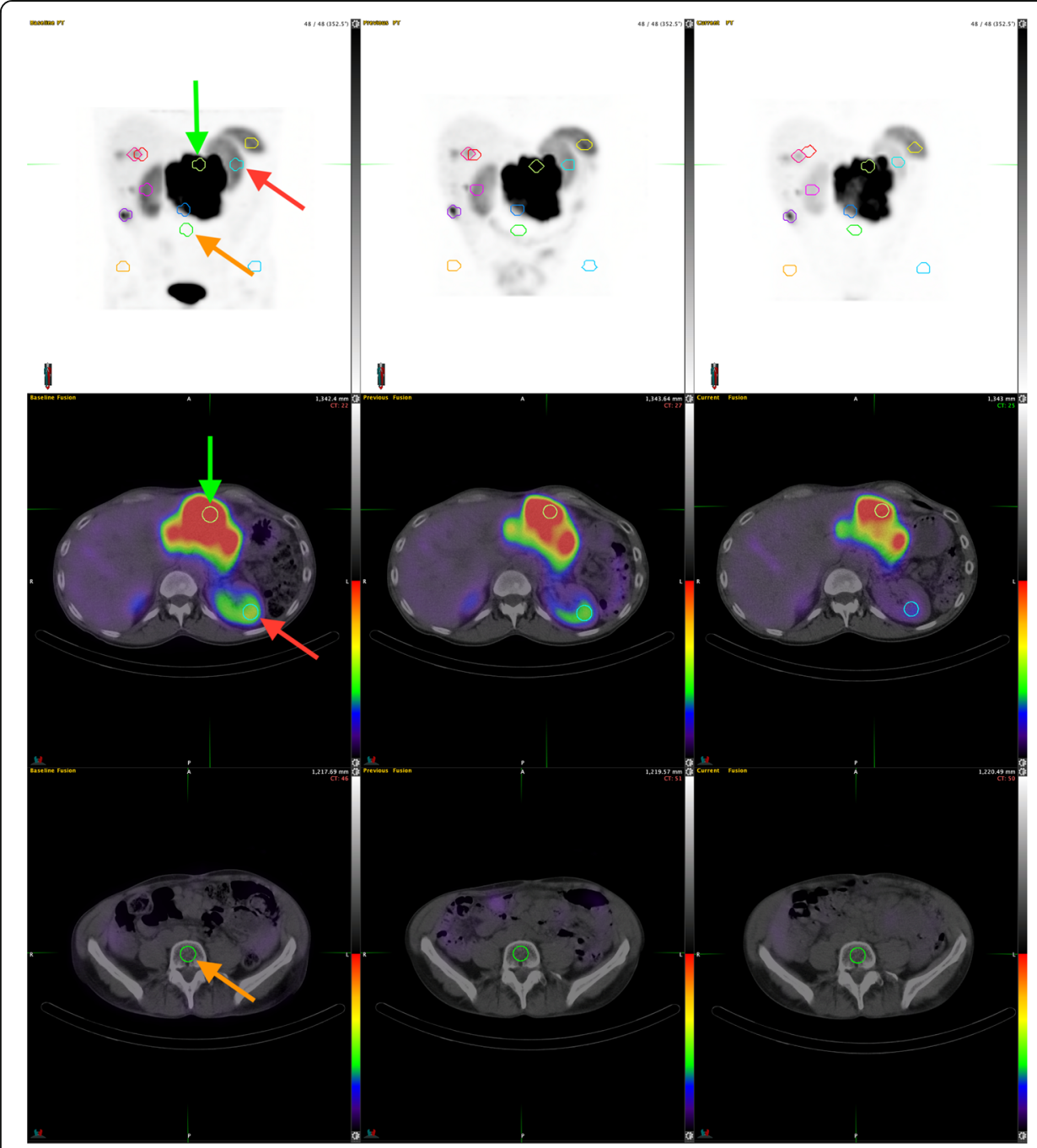

Fig. 1 Post-treatment serial QSPECT/CT was performed at (from left to right) 5, 24, and $70 \mathrm{~h}$ after a $22.0 \mathrm{GBq}{ }^{177} \mathrm{Lu}$-octreotate administration in a 55-year-old male with metastatic NET of unknown origin. Small volumes of interest (2-cm diameter) were placed over tissues of interest. Left kidney (red arrows), L5 bone marrow cavity (orange arrows), and dominant tumor (green arrows) VOls are pointed on anterior maximum intensity projections (top row) and selected transaxial fusion slices (mid and bottom rows). QSPECT images are normalized using an upper SUV threshold of 7. During this consolidation cycle, the personalized injected activity allowed the delivery of 6.1 Gy (6.0 Gy prescribed) to the kidney

As in our clinical practice, we routinely performed dosimetry based on the data acquired at these three time points (3TP), this approach constituted the reference method for the present analysis. In brief, at each time point, we sampled the activity concentration in tissues of interest (Fig. 1), including both kidneys (areas of representative parenchymal uptake), the BM (L4 and L5 vertebral bodies, or elsewhere when the latter were obviously affected by metastases), and the tumor having the most intense uptake (Tumor $\left.{ }_{\text {max }}\right)$, using $2-\mathrm{cm}\left(4.2 \mathrm{~cm}^{3}\right)$ spherical VOIs, as previously described [5, 6]. This was performed using either Hybrid Viewer (Hermes Medical Solutions, Stockholm, Sweden) or MIM Encore (MIM Software Inc., Cleveland, OH, USA) software. As previously described in [6], we also computed the total body retention for the purpose of computing the cross-dose component of the $\mathrm{BM}$ absorbed dose $\left(\mathrm{BM}_{\text {cross }}\right)$, which we 
added to the self-dose component $\left(\mathrm{BM}_{\text {self }}\right)$ to estimate the total $\mathrm{BM}$ absorbed dose $\left(\mathrm{BM}_{\text {total }}\right)$.

Based on these 3TP data, trapezoidal-monoexponential $\left(3 \mathrm{TP}_{\mathrm{TM}}\right)$ time-activity curves (TACs) were drawn using the following procedure (Fig. 2). For each organ/tumor, a constant mean SUV was assumed from the time of ${ }^{177} \mathrm{Lu}$-octreotate injection until the time of the day 0 scan (approximately $4 \mathrm{~h}$ ). This was followed by a linear (trapezoid) fit to the SUV corresponding to the day 0 and the day 1 scans. Then, a monoexponential curve was fit using the day 1 and day 3 data, resulting in an effective decay model being used from day 1 onwards (trapezoidal-monoexponential; $3 \mathrm{TP}_{\mathrm{TM}}$; Fig. 2). However, in cases when the day 3 SUV was higher than that corresponding to day 1, we assumed a linear SUV variation between days 1 and 3, followed by the physical decay of activity (i.e., $\lambda_{\text {biol }}=0, \lambda_{\text {eff }}=\lambda_{\text {phys }}$ ) from day 3 to infinity (trapezoidal-constant; $3 \mathrm{TP}_{\mathrm{TC}}$ ).

Then, the area under each TAC curve was integrated and multiplied by the appropriate activity concentration dose factors (ACDF). The values of these factors have been derived from OLINDA/EXM software data (Vanderbilt University, Nashville, TN, USA), as previously described [6]: $84 \mathrm{mGy} \cdot \mathrm{g} / \mathrm{MBq} / \mathrm{h}$ for Tumor $_{\max }$ and $87 \mathrm{mGy} \cdot \mathrm{g} /$ $\mathrm{MBq} / \mathrm{h}$ for kidneys and $\mathrm{BM}_{\text {self. For }} \mathrm{BM}_{\text {cross }}$, we integrated the total body activity over time and multiplied it by a dose factor of $1.09 \times 10^{-4} \mathrm{mGy} / \mathrm{MBq} / \mathrm{h}$ for males or $1.29 \times$ $10^{-4} \mathrm{mGy} / \mathrm{MBq} / \mathrm{h}$ for females, i.e., to account for their different gamma fraction of energy deposition from the whole body to the BM [6].

\section{Simplified dosimetry methods}

From our experience and as suggested by others, the day 0 data, although it captures the rapid kinetics of the radiopharmaceutical (which includes competing accumulation

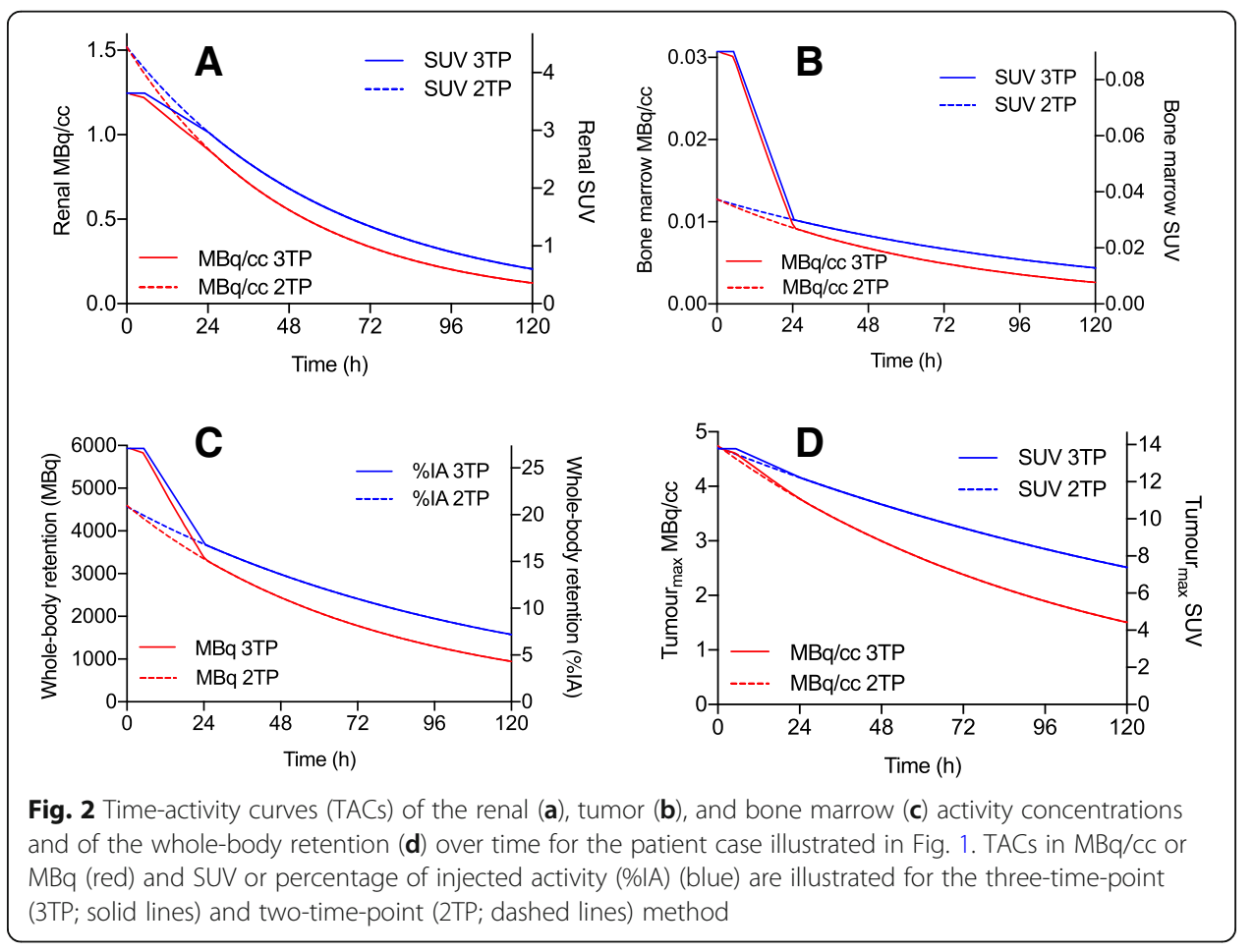


and rapid washout), contributes little to the area under the TAC, which is mostly determined by the slow washout kinetics and tends to follow a monoexponential decay be-

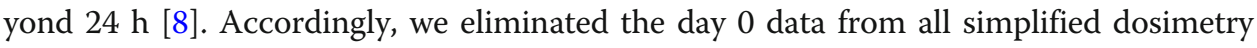
approaches. A total of five methods were investigated, as detailed below.

2TP: Two-time-point (2TP) dosimetry estimates were obtained using VOI data from day 1 to day 3 scans. From time of administration to infinity, monoexponential $\left(2 \mathrm{TP}_{\mathrm{M}}\right)$ effective decay was applied, except in cases of biological accumulation of activity, i.e., when the SUV of the tissue increased between day 1 and day 3. In such cases, we assumed a SUV equal to that of day $3 \mathrm{SUV}\left(\lambda_{\text {biol }}=0\right)$, from time of treatment administration to infinity and thus applied only physical decay $\left(\lambda_{\text {eff }}=\lambda_{\text {phys }} ; 2 \mathrm{TP}_{\mathrm{C}}\right)$. The $2 \mathrm{TP}$ method is the combination of $2 \mathrm{TP}_{\mathrm{M}}$ and $2 \mathrm{TP}_{\mathrm{C}}$.

$1 T P_{D 3}$ : As proposed by Hänscheid et al., we estimated doses using a single-time-point method based on the day 3 data $\left(1 \mathrm{TP}_{\mathrm{D} 3}\right)$ [8]. In this method, the activity concentration $(\mathrm{MBq} / \mathrm{cc})$ was multiplied by the time at which the day 3 scan was performed (h) and by $0.25 \mathrm{~Gy} \cdot \mathrm{g} / \mathrm{MBq} / \mathrm{h}$ (based on Eq. 8 in [8]). To compute $\mathrm{BM}_{\text {cross }}$, the total whole-body activity $(\mathrm{MBq})$ was multiplied by imaging time (h) and by $3.2 \times 10^{-7} \mathrm{~Gy} / \mathrm{MBq} / \mathrm{h}$ for males or $3.7 \times 10^{-7} \mathrm{~Gy} / \mathrm{MBq} / \mathrm{h}$ for females, i.e., the gamma fraction of energy deposition from the whole body to the $\mathrm{BM}$, multiplied by $0.25 \mathrm{~Gy} \cdot \mathrm{g} / \mathrm{MBq} / \mathrm{h}$ (from [8], as above), divided by $87 \mathrm{mGy} \cdot \mathrm{g} / \mathrm{MBq} / \mathrm{h}$ (ACDF of the $\mathrm{BM}$ and kidney).

$2 T P / 1 T P_{D 1}$ : We evaluated a hybrid dosimetry protocol based on the 2TP method for the first cycle, as described above, and employed a single scan on day 1 for the subsequent induction cycles. In this scenario, the absorbed dose to a given tissue during the second and the subsequent induction cycles was obtained by applying the monoexponential curve corresponding to the effective decay, as determined for this tissue during the first cycle, to the activity concentration observed on day 1 of the subsequent cycle.

$2 T P / 1 T P_{D 3}$ : This is the same as $2 \mathrm{TP} / 1 \mathrm{TP}_{\mathrm{D} 1}$, but the single scan on subsequent cycles was that performed on day 3.

2TP/NI: Similar to the two previous methods, this method was also based on 2TP scanning for the first cycle, but no imaging (NI) was performed for the subsequent cycles. For the latter, the absorbed dose per IA during the subsequent cycles was simply assumed to be equal to that delivered during the first cycle.

Cumulative renal, $\mathrm{BM}_{\text {total }}$, and Tumor ${ }_{\max }$ absorbed doses were compiled for all patients who received three or four induction cycles $(n=65)$. Per-cycle and cumulative doses resulting from each of the simplified dosimetry methods were compared with those obtained using the reference (3TP) method, and relative errors were calculated.

\section{Inter-observer variability}

For 60 induction cycles in 15 patients, the dosimetry analysis was performed independently by three observers having different backgrounds and purposely varied levels of experience in internal dosimetry. Observer 1 (M.D.P.), a certified endocrinologist, current PRRT Fellow and Ph.D. student, performed 258 of the 279 primary analyses described in this paper and, as such, accumulated the most experience with this dosimetry procedure. Observer 2 (F.A.) was a certified nuclear medicine physician and current $\mathrm{Nu}$ clear Oncology fellow who performed 21 primary analyses. Observer 3 (N.S.) was an 
M.D. student who was new to both nuclear medicine and dosimetry and who received only a short training. Relative errors of per-cycle and cumulative absorbed doses between each pair of observers were computed for the reference method (3TP) and the two most accurate simplified methods.

\section{Personalized ${ }^{177}$ Lu-octreotate activity prescription}

We previously derived a model based on the body surface area (BSA) and the estimated glomerular filtration rate (eGFR; according to the CKD-EPI Creatinine Equation [9]) to determine the personalized ${ }^{177} \mathrm{Lu}$-octreotate activity to be administered at the first cycle [6]. Using data from our entire cohort of 79 patients, we aimed to formulate a simpler prescription equation. To this end, we correlated the ratio of IA to the renal absorbed dose estimated from the first cycle (GBq/Gy, obtained by the 3TP or the 2TP methods) with the patient's weight, lean body weight (LBW), BSA, eGFR, and the products of eGFR with each of the three body size descriptors. Then, for each of these seven correlations, we performed a linear regression forced through the origin (eliminating the intercept) and calculated the relative errors of the predicted renal GBq/Gy using the slope of the linear regression.

We also compared the accuracy of predicting the renal GBq/Gy in any given non-initial cycle with that from the previous cycle or with the average renal GBq/Gy of the two previous cycles, as we have initially been doing in our P-PRRT trial [6].

\section{Statistical methods}

Data are presented as median and interdecile range or as mean \pm SD according to the data distribution using D'Agostino-Pearson omnibus normality test. Ranges are also reported. Pearson or Spearman correlations were used depending on the normality of the data. A difference was considered as statistically significant if the $P$ value was below 0.05. Correlations and linear regressions were performed using GraphPad Prism software (version 7, GraphPad Software Inc., La Jolla, CA, USA).

\section{Results}

\section{Accuracy of simplified dosimetry methods}

Tissue-specific effective half-lives derived from monoexponential fitting of the activity concentrations measured on days 1 and 3 are presented in Table 2. The per-cycle dosimetry results obtained with the 3TP and the 2TP methods are summarized in Table 3. For the kidney, there was only one patient case during which no biological elimination of activity between days 1 and 3 was observed. There were 30 such cases for the $\mathrm{BM}_{\text {self }}$ and 26 for Tumor $_{\max }$. In these cases, the $3 \mathrm{TP}_{\mathrm{TC}}$ and $2 \mathrm{TP}_{\mathrm{C}}$ methods were applied, while $3 \mathrm{TP}_{\mathrm{TM}}$ and $2 \mathrm{TP}_{\mathrm{M}}$ methods were used for all other cases. The 3TP and $2 \mathrm{TP}$ data (i.e., $3 \mathrm{TP}_{\mathrm{TM}}$ pooled with $3 \mathrm{TP}_{\mathrm{TC}}$, and $2 \mathrm{TP}_{\mathrm{M}}$ pooled with $2 \mathrm{TP}_{\mathrm{C}}$ ) were very highly correlated (Spearman $r>0.99, P<0.0001$ for all tissues). The median relative errors between the methods were small, particularly for the kidney and the tumor $(\leq 2 \%)$.

The results of applying the single-measurement method proposed by Hänscheid et al. [8] to our day 3 QSPECT uptake data $\left(1 \mathrm{TP}_{\mathrm{D} 3}\right)$ are shown in column 8 of Table 3 . We obtained the same median error for the kidney as Hänscheid (6\% at $72 \mathrm{~h})$ with a comparable interdecile range. Thus, our dosimetry results, based on tomographic data 
Table 2 Tissue-specific effective half-lives derived from activity concentration at day 1 and day 3, and absorbed doses per injected activity for the 3TP reference method $(n=279)$

\begin{tabular}{lll}
\hline & Effective half-life $(h)^{a}$ & Absorbed dose per injected activity (Gy/GBq) \\
\hline Kidney & $46.6[36.3-55.7](24.3-161.0)$ & $0.54[0.31-0.88](0.21-4.25)$ \\
Bone marrow $_{\text {self }}$ & $72.3[44.9-161.0](29.4-161.0)$ & $0.031[0.014-0.087](0.004-0.258)$ \\
Bone marrow $_{\text {cross }}{ }^{\mathrm{b}}$ & $66.9[50.3-91.6](24.6-121.6)$ & $0.0030[0.0016-0.0059](0.0005-0.0161)$ \\
Bone marrow $_{\text {total }}$ & - & $0.035[0.018-0.092](0.009-0.262)$ \\
Tumor $_{\text {max }}{ }^{c}$ & $100.9[60.0-158.4](27.7-161.0)$ & $3.8[1.0-8.6](0.1-32.0)$ \\
\hline
\end{tabular}

Data is presented as median [interdecile range] (range)

aln cases of biological accumulation of activity (kidney, $n=1$; bone marrow self, $n=30$; tumor max $_{1} n=26$ ), effective half-life was assumed to be equal to the physical half-life of ${ }^{177} \mathrm{Lu}$, i.e., $161 \mathrm{~h}$

${ }^{\mathrm{b}}$ Bone marrow cross-dose is derived from the gamma contribution of whole-body activity retention over time $c_{n=278}$

acquisition, validate this practical approach, which was devised using planar imaging data. Further, despite the different imaging techniques, we obtained a similar median effective half-life for the kidney (47 h, Table 2; vs. $51 \mathrm{~h}$ in [8]), although we observed a wider inter-patient variability. For Tumor ${ }_{\max }$, the $1 \mathrm{TP}_{\mathrm{D} 3}$ technique was slightly less accurate when applied to our data, but the range of errors was comparable.

Table 4 shows our results for the hybrid methods. In all cases, the 2TP method was applied in the first cycle. In this analysis, $2 \mathrm{TP} / 1 \mathrm{TP}_{\mathrm{D} 3}$ was found to be more accurate than both $2 \mathrm{TP} / 1 \mathrm{TP}_{\mathrm{D} 1}$ and $2 \mathrm{TP} / \mathrm{NI}$. The latter method yielded particularly inaccurate results for Tumor ${ }_{\max }$, due to the interference of therapeutic response. Please note that for all tissues, we obtained median errors closer to zero with $2 \mathrm{TP} / 1 \mathrm{TP}_{\mathrm{D} 3}$ than with $1 \mathrm{TP}_{\mathrm{D} 3}$ (Table 3). For the kidneys, among all the simplified dosimetry methods, $2 \mathrm{TP}$ was found to be the most accurate when compared to 3TP, on a per-cycle basis (Fig. 3).

As the aim of the induction course of our P-PRRT regime is to deliver a given prescribed renal absorbed dose, e.g., 23 Gy in patients without significant bone marrow or renal function impairment, we compared the accuracy of the simplified dosimetry methods to that of the 3TP method, for the assessment of the cumulative dosimetry in patients having completed at least three of the four intended induction cycles (Table 5). From all the simplified approaches, the 2TP method was by far the most accurate, in particular for the kidneys (Fig. 4). Using the latter, the cumulative renal and Tumor ${ }_{\max }$ absorbed dose for all patients agreed to within only $9 \%$ and $5 \%$, respectively, with the corresponding absorbed doses derived from 3TP scanning (Table 5) confirming the small influence of the day 0 scan and early kinetics on the precision of dosimetry estimates. Even the total BM dose was quite accurately estimated using the 2TP protocol. When compared with 2TP, median errors increased, and error ranges widened for all 1TP-based techniques and even more so if no imaging was done on subsequent cycles.

\section{Inter-observer variability}

Table 6 illustrates the inter-observer variability of the per-cycle and cumulative renal, $\mathrm{BM}_{\text {total }}$, and $\mathrm{Tumor}_{\max }$ absorbed doses assessed independently by the three observers in 15 patients (60 cycles) using three methods: $3 \mathrm{TP}, 2 \mathrm{TP}$, and $2 \mathrm{TP} / 1 \mathrm{TP} \mathrm{D}_{\mathrm{D}}$. There was an excellent inter-observer agreement between all observers for the kidney using the three methods, the best agreement being for the cumulative renal dose estimated using the 3TP and 2TP methods. The span of errors was larger for Tumor $_{\max }$, owing it to 


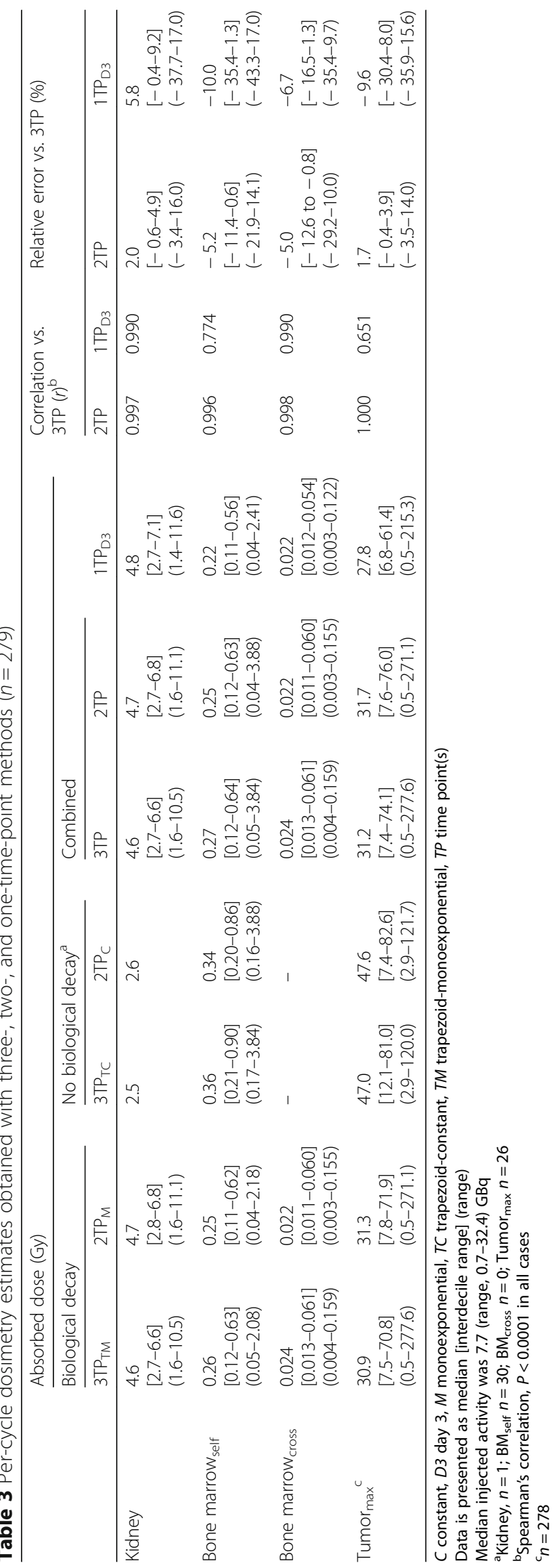




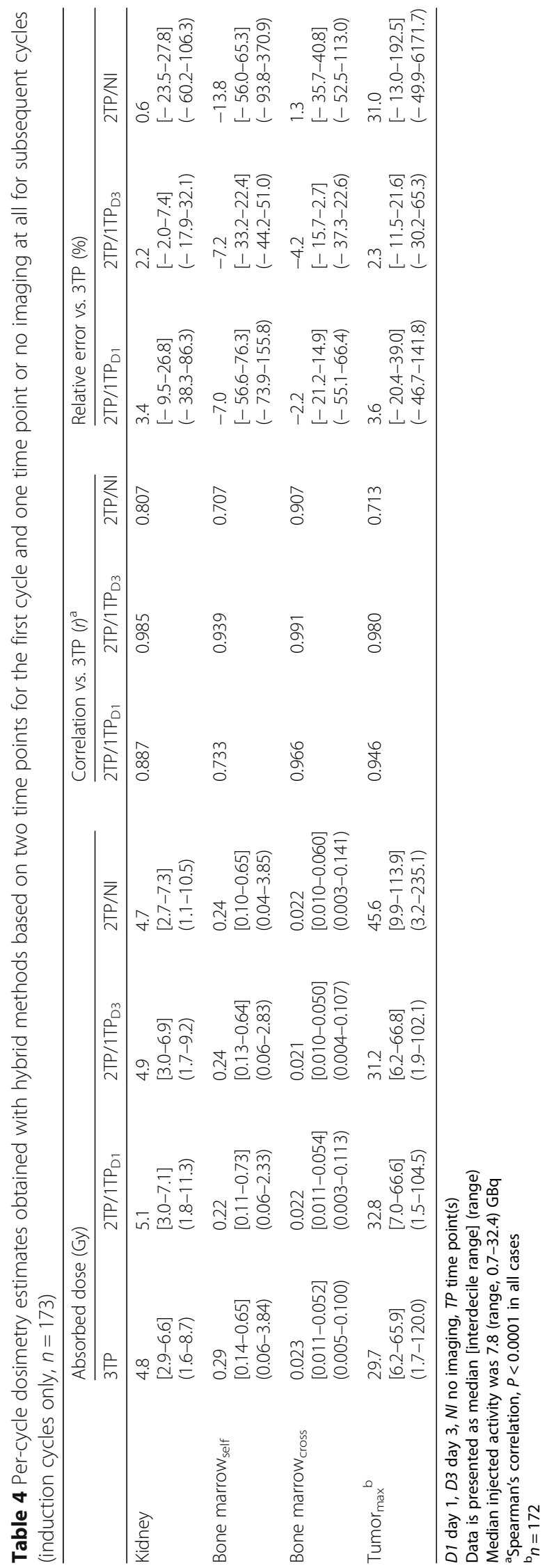




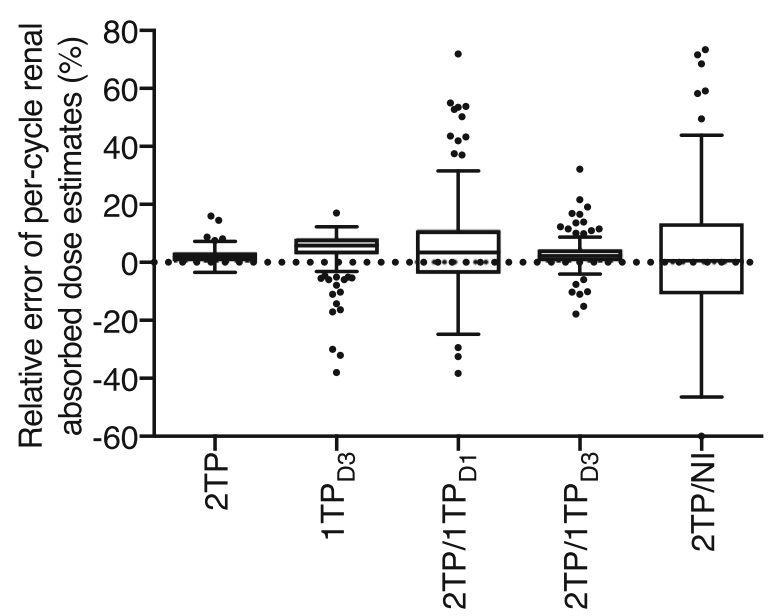

Fig. 3 Comparison of the relative errors of per-cycle renal absorbed dose estimates obtained by the simplified methods relative to the three-time-point (3TP) method. Boxes represent the interquartile range, and whiskers the interdecile range (2TP and $1 \mathrm{TP}_{\mathrm{D} 3}, n=279 ; 2 \mathrm{TP} / 1 \mathrm{TP}_{\mathrm{D} 1}, 2 \mathrm{TP} / 1 \mathrm{TP} \mathrm{D}_{\mathrm{D}}$, and $2 \mathrm{TP} / \mathrm{NI}, n=173$ )

variations in the precise placement of the VOI over the most intense region of the dominant lesion. For both $\mathrm{BM}_{\text {total }}$ and Tumor ${ }_{\max }$, there was a trend towards a lesser inter-observer agreement when the least experienced observer (observer 3) was involved. $\mathrm{BM}_{\text {total }}$ reproducibility suffered from the low-level and noisy uptake data in the $\mathrm{BM}$ compartment, and consequently $\mathrm{BM}_{\text {self }}$, the dominant component of $\mathrm{BM}_{\text {total }}$, was more sensitive to the position of the VOI than were the absorbed doses of the other tissues of interest. Nevertheless, the inter-observer agreement on the cumulative $\mathrm{BM}_{\text {total }}$ dose was fair.

\section{Accuracy of activity prescription at first and subsequent cycles}

Correlations and linear regression slopes between the body size descriptors, the eGFR, or their products vs. the IA per renal absorbed dose at the first induction cycle are reported in Table 7. The strongest correlations were found when using either eGFR - LBW or eGFR · BSA (Fig. 5) as predictors of renal GBq/Gy, and both seem equally appropriate for personalized IA prescription at the first cycle (Fig. 6). We therefore elected to continue using eGFR and BSA for determining IA at the first cycle and updated our initial formula (found in [6]) with this simpler equation:

$$
\begin{aligned}
\text { Personalized AI }(\mathrm{GBq})= & K \cdot \mathrm{eGFR}\left(\mathrm{mL} / \mathrm{min} / 1.73 \mathrm{~m}^{2}\right) \cdot \mathrm{BSA}\left(\mathrm{m}^{2}\right) \\
& \cdot \text { Prescribed renal absorbed dose }(\mathrm{Gy})
\end{aligned}
$$

where $K=0.012 \mathrm{GBq} \cdot \mathrm{min} \cdot 1.73 / \mathrm{mL} / \mathrm{Gy}$, i.e., the slope of the linear regression (Fig. 5).

For the subsequent cycles, the median error of the renal GBq/Gy relative to that of the previous cycle was $-0.7 \%$ (interdecile range, -21.0 to $20.2 \%$; range, -49.1 to $54.0 \% ; n=194$ ) for the 3TP method, $-0.8 \%$ (interdecile range, -20.4 to $19.0 \%$; range, -49.4 to $50.2 \% ; n=194$ ) for the $2 \mathrm{TP}$ method, and $-0.3 \%$ (interdecile range, -20.5 to $18.6 \%$; range, -40.2 to $87.4 \% ; n=168)$ for the $2 \mathrm{TP} / 1 \mathrm{TP}_{\mathrm{D} 3}$ method. When in the analysis of the third and fourth induction cycle the average of the renal GBq/Gy of the two prior cycles was used, the resulting errors were $-0.9 \%$ (interdecile range, $-21.3-19.1 \%$; range, $-49.9-54.4 \% ; n=192$ ), $-0.6 \%$ (interdecile range, $-21.4-18.6 \%$; range, $-48.5-$ 


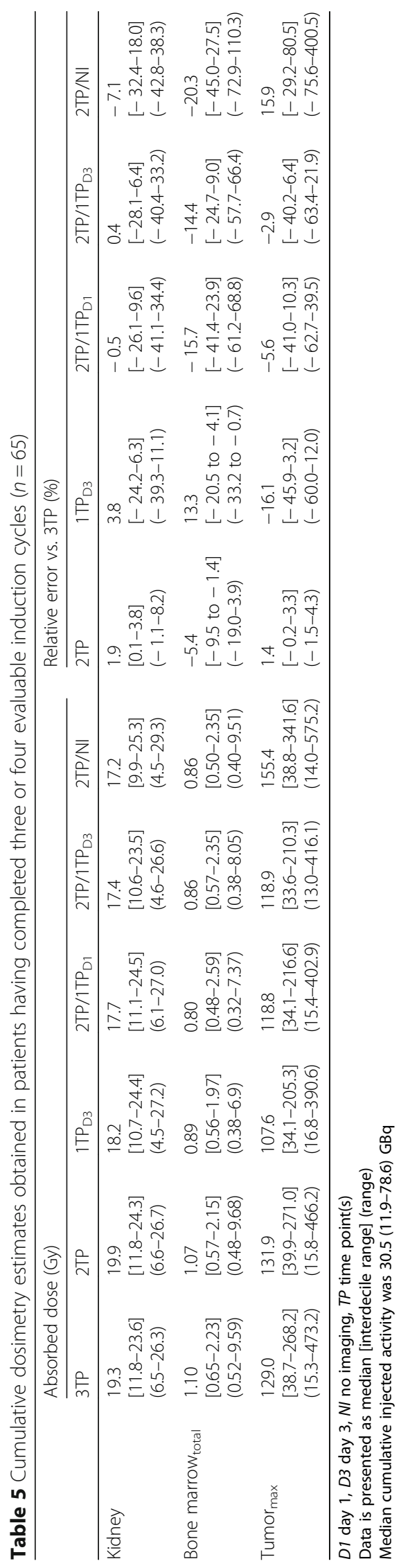




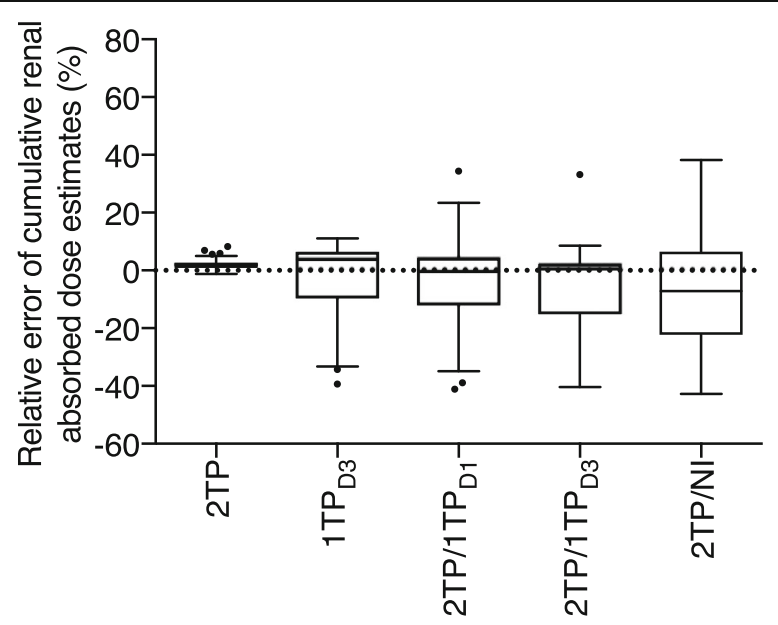

Fig. 4 Comparison of the relative errors of per-induction course cumulative renal absorbed dose estimates obtained by the simplified methods relative to the three-time-point (3TP) method. Boxes represent the interquartile range, and whiskers the interdecile range $(n=65)$

$54.5 \% ; n=192$ ) and $-0.6 \%$ (interdecile range, $-19.8-19.6 \%$; range, $-45.7-75.3 \% ; n=$ 166), respectively. Hence, unlike in our initial P-PRRT protocol, these results convinced us that averaging the renal GBq/Gy from the two prior cycles (instead of just one) does not significantly improve the precision of the IA prescription. The IA prescription for the subsequent cycle has been updated in our P-PRRT protocol as follows:

Personalized IA $(\mathrm{GBq})=$ Prior cycle IA per renal dose $(\mathrm{GBq} / \mathrm{Gy})$ - Prescribed renal dose (Gy)

\section{Discussion}

The widely adopted one-size-fits-all PRRT protocol, i.e., four induction cycles of 7.4 GBq ${ }^{177} \mathrm{Lu}$-octreotate, has been initially devised in 2001 based on the dosimetry data from only five patients [3]. Since that time, dosimetry has not been routinely performed in most centers (including for PRRT administered in the NETTER-1 trial [2]). This fixed IA regime is known to yield highly variable absorbed doses to critical organs, but because these fall well below conservative safety thresholds (e.g., 23 Gy for the kidney) in the vast majority of patients, this confers to PRRT a very favorable safety profile $[1,2]$. In parallel, current cure rates are marginal, suggesting that most patients are being undertreated with the empiric PRRT regime [1,2].

Escalating tumor absorbed dose could potentially improve the efficacy of PRRT, although realistically, this cannot be done through a conventional empiric IA escalation without compromising the excellent safety record of PRRT. To optimize tumor irradiation at the patient level, we and others have proposed to optimize the renal absorbed dose by either personalizing the IA per cycle or the number of induction cycles $[4,6]$. These two approaches resulted in increased cumulative IA and tumor absorbed dose in the majority of patients $[4,6]$. Further, when administering personalized IA, our preliminary results suggest a similar short-term side effect and toxicity profile as those observed when using the empiric PRRT regime [10]. Although our outcome data is not yet mature enough to document significantly improved clinical outcomes, nevertheless, 
Table 6 Inter-observer variability of dosimetry estimates in 60 induction cycles received by 15 patients

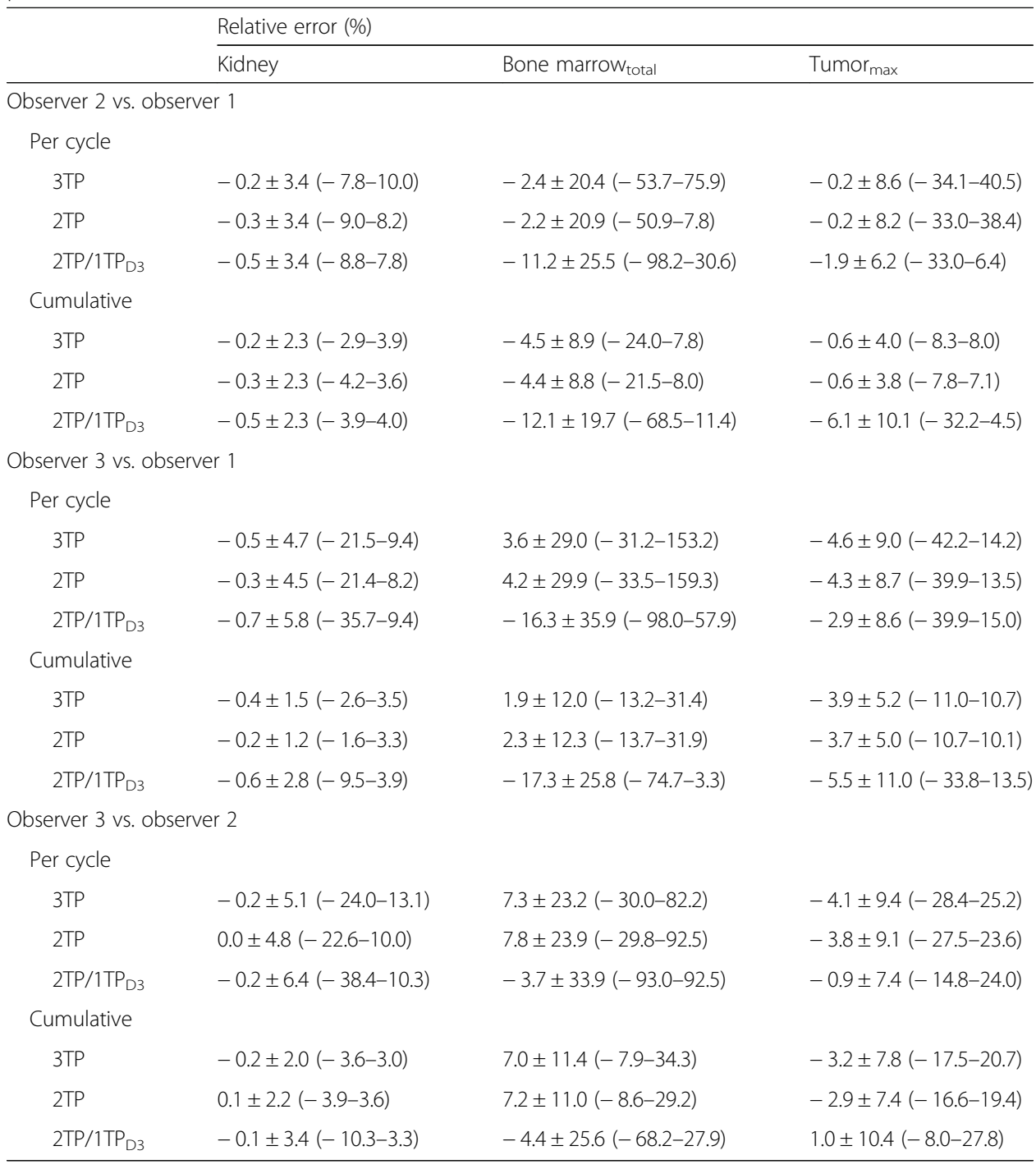

D3 day 3, TP time point(s)

Data is presented as mean \pm SD (range)

we believe that personalized radionuclide therapy is more faithful to the principles of radiation oncology, where the absorbed doses are prescribed and monitored. In internal radiotherapy, this could be done through IA personalization and routine dosimetry.

Despite the fact that our imaging protocol did not include a late time point, we obtained similar median renal absorbed doses per IA (median, $0.54 \mathrm{~Gy} / \mathrm{GBq}$ ) to those observed by Sandstrom et al. (medians, 0.62 and $0.59 \mathrm{~Gy} / \mathrm{GBq}$ for the right and the left kidneys, respectively), who also used QSPECT and small-VOI sampling, but scanned patients until day 7 [5]. The concordance between our results is consistent with the fact that the renal activity concentration decays moxoexponentially after $24 \mathrm{~h}$, as demonstrated by Handshied et al. [8].

The median BM absorbed dose we obtained when using our QSPECT-based method $(0.035 \mathrm{~Gy} / \mathrm{GBq})$ is well within the range of estimates published by others using various techniques based on imaging, blood, and urine sampling $[5,11]$. Furthermore, this 
Table 7 Correlation between body size predictors, eGFR, and the IA per renal absorbed dose (GBq/Gy) at the first induction cycle $(n=77)$

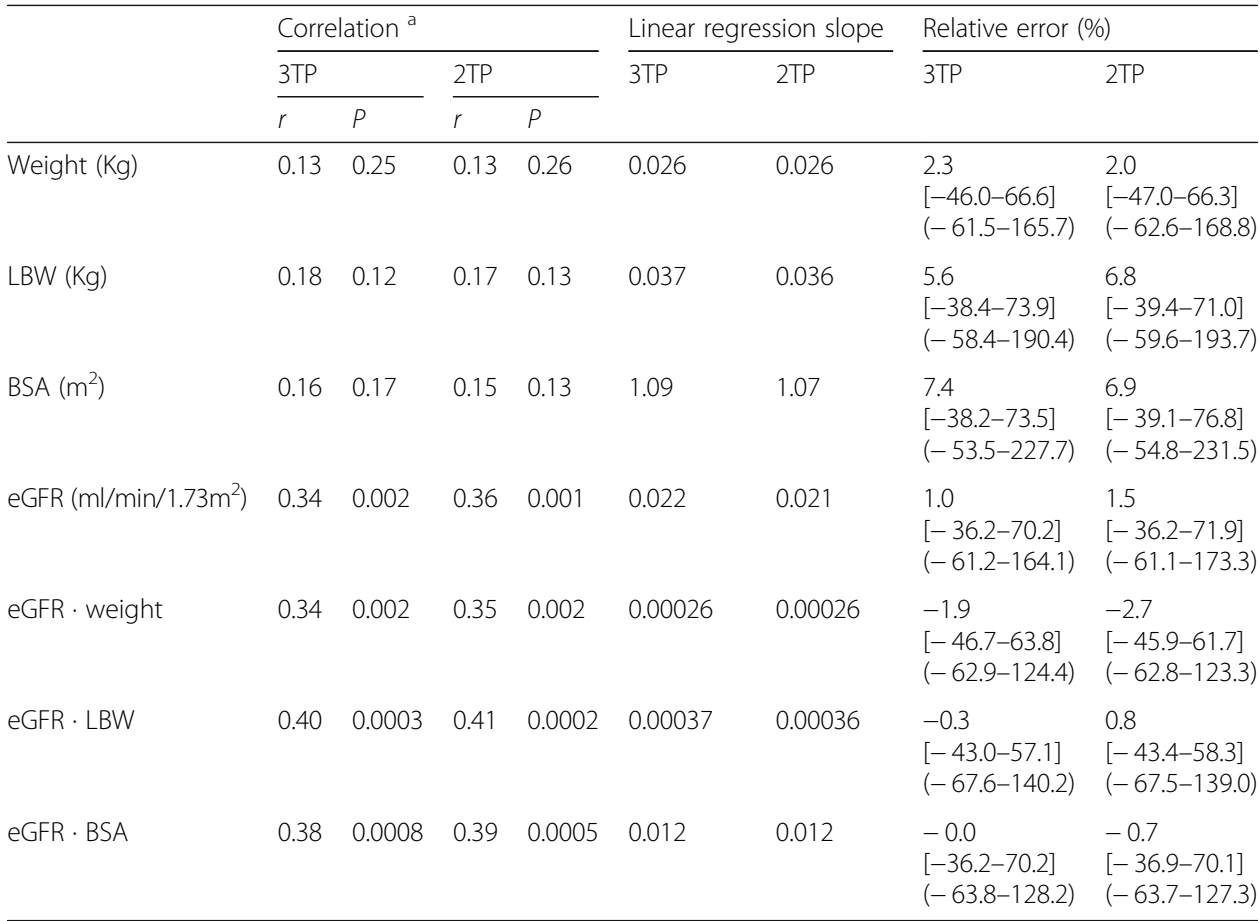

$B S A$ body surface area, eGFR estimated glomerular filtration rate, $L B W$ lean body weight, TP time points Relative error data is presented as median [interdecile range] (range) ${ }^{\text {a }}$ Spearman's correlation

result is particularly close to the mean BM absorbed dose reported by Svensson et al. (0.027 Gy/GBq), which was derived from imaging data only and included a later time point $[12,13]$. The correlation between QSPECT-based BM absorbed dose estimates and subacute thrombocytopenia provides initial clinical validation of our technique [6]. However, in patients with bone metastases, the BM dosimetry estimates may

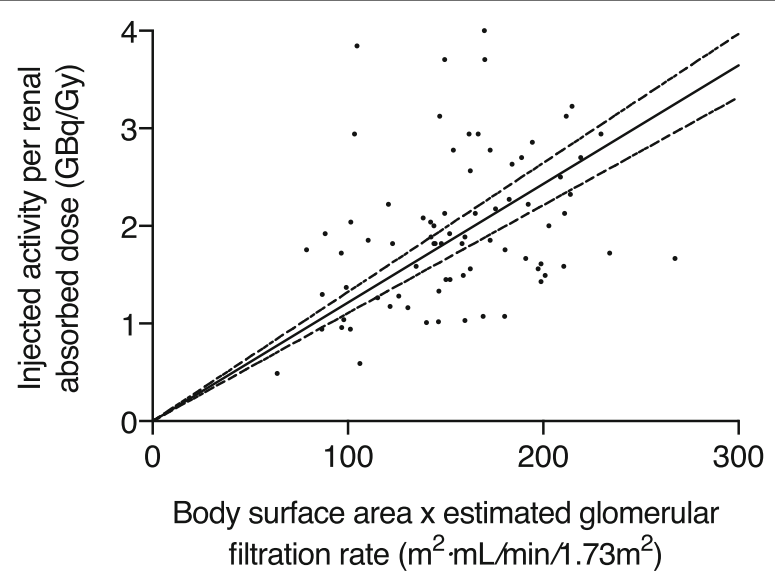

Fig. 5 Injected activity per renal absorbed dose at the first cycle vs. the product of body surface area and estimated glomerular filtration rate $(n=77)$. There was a moderate correlation between variables (Spearman $r=0.39, P=0.0005$ ). The slope of the linear regression curve forced through origin (solid line; $95 \%$ confidence interval, dashed lines), which was $0.012 \mathrm{GBq} \cdot \min \times 1.73 / \mathrm{mL} / \mathrm{Gy}$, is to be used to adjust the injected activity at the first cycle in a personalized PRRT protocol 


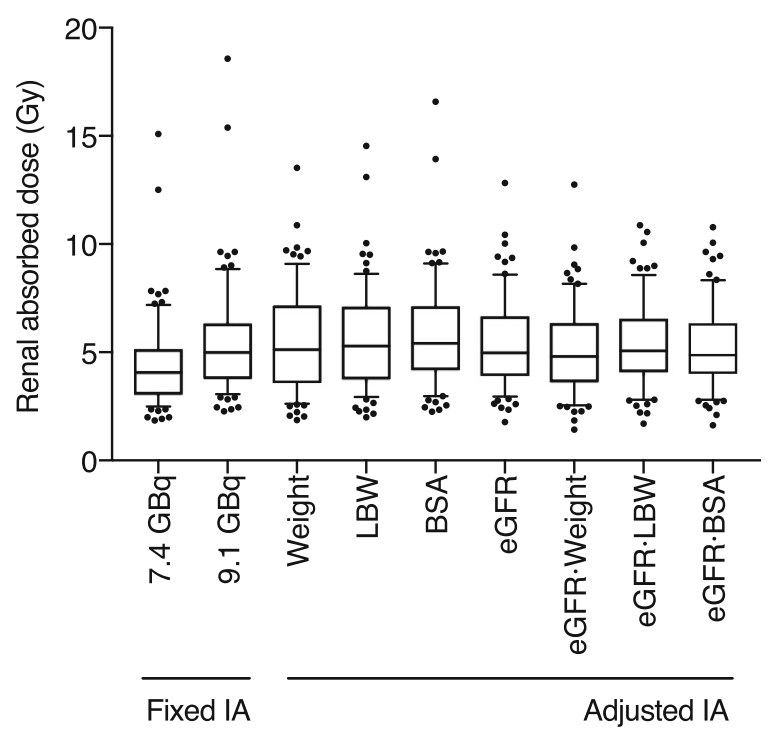

Fig. 6 Comparison of renal absorbed dose delivered during the first cycle of fixed injected activity (IA) vs. personalized PRRT regimes $(n=77)$. In the latter, the prescribed renal absorbed dose is 5 Gy and the IA is adjusted based on weight, lean body weight (LBW), body surface area (BSA), estimated glomerular filtration rate (eGFR), or the product of eGFR and of a body size descriptor. For comparison, a fixed IA of $9.1 \mathrm{GBq}$ would yield a median renal absorbed renal absorbed dose of 5 Gy

be less reliable, as even if obvious bone metastases were avoided when placing the BM VOIs, we cannot rule out the influence of non-apparent micrometastases or diffuse BM infiltration.

Dosimetry is an essential component of P-PRRT but is often perceived by the medical community as being too complex, or by the physics community as not accurate enough. However, SPECT/CT cameras are now widely available, and simple ${ }^{177} \mathrm{Lu}$ calibration methods have been proposed [7, 14], facilitating implementation of individualized dosimetry based on QSPECT in the clinics. Performing dosimetry calculation based on simplified activity concentration sampling methods, such as the small-VOI method used in this study, is more practical to perform than the full organ segmentation while yielding similar results and is more accurate than planar imaging-based dosimetry [15]. For these reasons, we have routinely been performing dosimetry using a 3TP QSPECT scanning schedule along with the small-VOI sampling. But still, many would see dosimetry as resource-consuming. This opinion would be based on the general beliefs that a minimum of three measurements are necessary [16] or that the scanning protocol must include late time points, such as up to 4 to 7 days [17]. Such requirements tend to increase both the clinical burden and the patient inconvenience when performing dosimetry, and as such constitute barriers to its wide clinical adoption. Conversely, simplified dosimetry approaches having a clinically relevant level of accuracy could facilitate making dosimetry a standard of care, not just for monitoring purposes, but also for personalizing radionuclide therapy. To overcome the issues discussed above, the primary objective of this study was to further simplify our dosimetry methods.

The 2TP method offers an excellent accuracy for both the per-cycle and the cumulative absorbed dose estimates relative to the 3TP method, in particular for the kidney and the tumor, which convinced us to abandon the day 0 scan. Further, our 
results validated the $1 \mathrm{TP}_{\mathrm{D} 3}$ technique proposed by Hänscheid and co-workers [8]. While they advocate scanning on day 4 to achieve the best accuracy for both the kidney and the tumor, scanning on day 3 is considered more practical in our setting, offers about the same accuracy for the kidney dosimetry and a very reasonable accuracy for the tumor. This $1 \mathrm{TP}_{\mathrm{D} 3}$ method is an appealing alternative to 2TP, although accuracy could be slightly improved, at least for the kidney and Tumor $_{\max }$, by simply adding a second imaging time point during the first cycle and then applying the effective decay constant to the one-time samples during subsequent cycles (2TP/1TP). This hybrid method is more accurate when, for the non-initial cycles, the imaging is performed on day $3\left(2 \mathrm{TP} / 1 \mathrm{TP}_{\mathrm{D} 3}\right)$ rather than on day $1\left(2 \mathrm{TP} / 1 \mathrm{TP}_{\mathrm{D} 1}\right)$. This is likely because, in individual patients, day 3 measurements are better correlated with the integrated TACs (i.e., absorbed doses) than are those performed on day 1 and, as such, are less sensitive to small intra-patient cycle-to-cycle differences in tissue uptake and kinetics [8]. However, since in parallel we alter the IA prescription based on renal dosimetry, we prefer pursuing our P-PRRT program with the 2TP protocol, which offers, in our opinion, the best balance between high accuracy and practicality. Importantly, we would not recommend not imaging patients at subsequent cycles and extrapolating dosimetry from the first cycle (2TP/NI), assuming constant Gy/GBq in tissues (i.e., completely ignoring any cycle-to-cycle difference in uptake or kinetics), as this approach causes the uncertainty of the resulting absorbed dose estimates to increase, in particular for tumor, which can be affected by the therapeutic response. The clinical burden of the 2TP schedule in terms of the camera and personnel time is reasonable and comparable to that of performing an ${ }^{111}$ In-octreotide scan. Furthermore, performing the last scan on the third day limits the inconvenience for the out-of-city patients.

A very good inter-observer agreement has already been reported for renal dosimetry, with median errors of less than 5\% for the small-VOI dosimetry method [17]. Our results confirm these observations. Of note, we intentionally chose observers with different backgrounds and have shown that even dosimetry estimates from our novice observer (first-year medical student) were well in agreement with those from more experienced observers, suggesting that a reasonably reproducible activity concentration sampling technique is easily attainable with a relatively short training. Also, the whole processing of one patient case, including VOI drawing and data transfer to the spreadsheet or database, can be performed in about 15 to $20 \mathrm{~min}$. We are contemplating to train nuclear medicine technologists to perform PRRT dosimetry under medical supervision, eventually making them sub-specialized as nuclear dosimetrists.

Finally, we revisited our personalized IA prescription scheme for our P-PRRT protocol. For the first cycle, we derived a simpler equation to determine the personalized IA than the one we initially suggested [6]. The latter is still based on the product of eGFR and BSA, but eGFR - LBW would have provided a similar level of predictive accuracy. We acknowledge that this accuracy is at most moderate and comparable to that of an initial fixed IA in terms of interquartile or interdecile range (Fig. 6). However, the main advantage of personalizing the first cycle IA is to avoid extreme cases of overdosing, such as delivery 18 Gy to the kidney when administering a fixed IA of $9.1 \mathrm{GBq}$ to every patient (Fig. 6). Rather, personalizing IA could limit the renal absorbed dose to $11 \mathrm{~Gy}$, for the same median of $5 \mathrm{~Gy}$. When 
${ }^{68}$ Ga-octreotate PET will be routinely performed in all our PRRT patients, we will explore adding the pre-treatment tumor sink effect analysis into the prescription scheme, which could potentially improve the predictive accuracy of the model [18].

\section{Conclusions}

We propose a ${ }^{177} \mathrm{Lu}$ dosimetry protocol based on two-time-point QSPECT imaging and the small-VOI sampling, which yields accurate dosimetry results, particularly for the kidney and the tumor, with a very high inter-observer reproducibility. Performing the last QSPECT/CT scan no later than on the third day post-PRRT increases patient convenience, particularly for the out-of-city patients who travel to receive PRRT. Pragmatic ${ }^{177} \mathrm{Lu}$ dosimetry methods could facilitate the practice of personalized radionuclide therapies, including the rapidly emerging prostate-specific membrane antigen radioligand therapy.

\section{Abbreviations}

ACDF: Activity concentration dose factor; BSA: Body surface area; CT: Computed tomography; eGFR: Estimated glomerular filtration rate; IA: Injected activity; LBW: Lean body weight; NET(s): Neuroendocrine tumor(s); NI: No imaging; PET: Positron emission tomography; P-PRRT: Personalized PRRT; PRRT: Peptide receptor radionuclide therapy; QSPECT: Quantitative SPECT; SPECT: Single-photon emission computed tomography; SUV: Standardized uptake value;

TAC(s): Time-activity curve(s); TP: Time point; VOI(s): Volume(s) of interest

\section{Acknowledgements}

We are grateful to the nurses and nuclear medicine technologists at the CHU de Québec-Université Laval who provided care to PRRT patients, as well as to Marc Bazin, Ph.D., for his help with the writing of this manuscript.

\section{Funding}

M.D.P. is supported by a Merit Scholarship for Foreign Students from the Ministère de l'éducation et de l'enseignement supérieur du Québec. This work was funded by the Canadian Institutes of Health Research (CIHR) operating grant MOP142233 to J.M.B.

\section{Availability of data and materials}

Please contact the corresponding author, Jean-Mathieu Beauregard (jean-mathieu.beauregard@chudequebec.ca), for the data used in this manuscript.

\section{Authors' contributions}

MDP participated in the design of the analysis, collected and analyzed the data, and drafted the manuscript. FA and NS performed dosimetry analyses. FAB and JMB were responsible for the good conduct of the clinical study and data acquisition. WZ and AC participated in the design of the analysis and contributed to the manuscript editing. JMB designed the analysis, supervised the project, analyzed the data, and edited the manuscript. All authors read and approved the final manuscript.

\section{Ethics approval and consent to participate}

All procedures performed in studies involving human participants were in accordance with the ethical standards of the institutional and/or national research committee and with the 1964 Helsinki declaration and its later amendments or comparable ethical standards. Specifically, for the 23 patients who received only empiric PRRT until March 2016, the requirement for consent was waived due to the retrospective nature of the analysis. All other patients were enrolled in our P-PRRT trial (NCT02754297) and gave informed consent to participate.

\section{Consent for publication}

All authors read the manuscript and consented for its publication.

\section{Competing interests}

The authors declare that they have no competing interests.

\section{Publisher's Note}

Springer Nature remains neutral with regard to jurisdictional claims in published maps and institutional affiliations.

\section{Author details}

${ }^{1}$ Department of Radiology and Nuclear Medicine and Cancer Research Center, Université Laval, Quebec City, Canada. ${ }^{2}$ Department of Medical Imaging and Oncology Branch of CHU de Québec Research Center, CHU de Québec Université Laval, 11 côte du Palais, Quebec City, QC G1R 2J6, Canada. ${ }^{3}$ Medical Imaging Research Group, University of British Columbia, Vancouver, Canada. ${ }^{4}$ Department of Physics and Astronomy, University of British Columbia, Vancouver, Canada. 
Received: 4 April 2018 Accepted: 1 August 2018

Published online: 15 October 2018

\section{References}

1. Kwekkeboom DJ, de Herder WW, Kam BL, van Eijck CH, van Essen M, Kooij PP, et al. Treatment with the radiolabeled somatostatin analog $\left[{ }^{177}\right.$ Lu-DOTA ${ }^{0}$, Tyr $\left.^{3}\right]$ octreotate: toxicity, efficacy, and survival. J Clin Oncol. 2008; 26(13):2124-30. https://doi.org/10.1200/JCO.2007.15.2553.

2. Strosberg J, El-Haddad G, Wolin E, Hendifar A, Yao J, Chasen B, et al. Phase 3 trial of ${ }^{177}$ Lu-Dotatate for midgut neuroendocrine tumors. N Engl J Med. 2017;376(2):125-35. https://doi.org/10.1056/NEJMoa1607427.

3. Kwekkeboom DJ, Bakker WH, Kooij PP, Konijnenberg MW, Srinivasan A, Erion JL, et al. [177 Lu-DOTA ${ }^{0}$ yrr $\left.^{3}\right]$ octreotate: comparison with [111 In-DTPA ${ }^{0}$ octreotide in patients. Eur J Nucl Med. 2001;28(9):1319-25.

4. Sundlov A, Sjogreen-Gleisner K, Svensson J, Ljungberg M, Olsson T, Bernhardt P, et al. Individualised ${ }^{177} \mathrm{Lu}-$ DOTATATE treatment of neuroendocrine tumours based on kidney dosimetry. Eur J Nucl Med Mol Imaging. 2017; 44(9):1480-9. https://doi.org/10.1007/s00259-017-3678-4.

5. Sandstrom M, Garske-Roman U, Granberg D, Johansson S, Widstrom C, Eriksson B, et al. Individualized dosimetry of kidney and bone marrow in patients undergoing ${ }^{177}$ Lu-DOTA-octreotate treatment. J Nucl Med. 2013;54(1):3341. https://doi.org/10.2967/jnumed.112.107524

6. Del Prete M, Buteau FA, Beauregard JM. Personalized ${ }^{177} \mathrm{Lu}$-octreotate peptide receptor radionuclide therapy of neuroendocrine tumours: a simulation study. Eur J Nucl Med Mol Imaging. 2017;44(9):1490-500. https://doi.org/10 1007/s00259-017-3688-2.

7. Beauregard JM, Hofman MS, Pereira JM, Eu P, Hicks RJ. Quantitative ${ }^{177}$ Lu SPECT (QSPECT) imaging using a commercially available SPECT/CT system. Cancer Imaging. 2011;11:56-66. https:/doi.org/10.1102/1470-7330.2011.0012

8. Hanscheid H, Lapa C, Buck AK, Lassmann M, Werner RA. Dose mapping after endoradiotherapy with ${ }^{177} \mathrm{Lu}$ DOTATATE/DOTATOC by a single measurement after 4 days. J Nucl Med. 2018;59(1):75-81. https://doi.org/10. 2967/jnumed.117.193706.

9. Levey AS, Stevens LA, Schmid CH, Zhang YL, Castro AF 3rd, Feldman HI, et al. A new equation to estimate glomerular filtration rate. Ann Intern Med. 2009;150(9):604-12.

10. Del Prete M, Buteau FA, Beaulieu A, Beauregard JM. Personalized ${ }^{177}$ Lu-octreotate peptide receptor radionuclide therapy of neuroendocrine tumors: initial dosimetry and safety results of the P-PRRT trial. J Nucl Med. 2017; 58(Suppl. 1):242.

11. Cremonesi M, Botta F, Di Dia A, Ferrari M, Bodei L, De Cicco C, et al. Dosimetry for treatment with radiolabelled somatostatin analogues. A review. Q J Nucl Med Mol Imaging. 2010;54(1):37-51.

12. Svensson J, Rydén T, Hagmarker L, Hemmingsson J, Wängberg B, Bernhardt P. A novel planar image-based method for bone marrow dosimetry in ${ }^{177}$ Lu-DOTATATE treatment correlates with haematological toxicity. EJNMMI Physics. 2016;3:21. https://doi.org/10.1186/s40658-016-0157-0.

13. Hagmarker L, Svensson J, Rydén T, Gjertsson P, Bernhardt P. Segmentation of whole-body images into two compartments in model for bone marrow dosimetry increases the correlation with hematological response in 177Lu-DOTATATE treatments. Cancer Biother Radiopharm. 2017;32(9):335-43. https://doi.org/10.1089/cbr.2017.2317.

14. Uribe CF, Esquinas PL, Tanguay J, Gonzalez M, Gaudin E, Beauregard JM, et al. Accuracy of ${ }^{177}$ Lu activity quantification in SPECT imaging: a phantom study. EJNMMI Phys. 2017;4(1):2. https:/doi.org/10.1186/s40658-016-0170-3.

15. Sandstrom M, Garske U, Granberg D, Sundin A, Lundqvist H. Individualized dosimetry in patients undergoing therapy with ${ }^{177}$ Lu-DOTA-D-Phe ${ }^{1}$-Tyr ${ }^{3}$-octreotate. Eur J Nucl Med Mol Imaging. 2010;37(2):212-25. https://doi.org/ 10.1007/s00259-009-1216-8

16. Lassmann M, Chiesa C, Flux G, Bardies M, Committee ED. EANM dosimetry committee guidance document: good practice of clinical dosimetry reporting. Eur J Nucl Med Mol Imaging. 2011;38(1):192-200. https://doi.org/10.1007/ s00259-010-1549-3.

17. Sandstrom M, llan E, Karlberg A, Johansson S, Freedman N, Garske-Roman U. Method dependence, observer variability and kidney volumes in radiation dosimetry of ${ }^{177}$ Lu-DOTATATE therapy in patients with neuroendocrine tumours. EJNMMI Phys. 2015;2(1):24. https://doi.org/10.1186/s40658-015-0127-y.

18. Beauregard JM, Hofman MS, Kong G, Hicks RJ. The tumour sink effect on the biodistribution of ${ }^{68} \mathrm{Ga}$-DOTAoctreotate: implications for peptide receptor radionuclide therapy. Eur J Nucl Med Mol Imaging. 2012;39(1):50-6. https://doi.org/10.1007/s00259-011-1937-3.

\section{Submit your manuscript to a SpringerOpen ${ }^{\circ}$ journal and benefit from:}

- Convenient online submission

- Rigorous peer review

- Open access: articles freely available online

- High visibility within the field

- Retaining the copyright to your article

Submit your next manuscript at $\boldsymbol{\nabla}$ springeropen.com 